\title{
ON ASSOCIATIVE COMPOSITIONS IN FINITE NILPOTENT GROUPS
}

\author{
NAGAYOSI IWAHORI and AKIRA HATTORI
}

Let

$$
f(X, Y)=X^{m_{1}} Y^{n_{1}} \ldots X^{m_{r}} Y^{n_{r}}
$$

be a word in two variables $X, Y$, i.e. an element in the free group $F_{2}$ on two generators $X, Y$. Let us say that $f$ defines an associative composition for a group $G$ if for arbitrary elements $a, b, c$ in $G$ we have

$$
(a \circ b) \circ c=a \circ(b \circ c)
$$

where $a \circ b$ is defined by

$$
a \circ b=f(a, b) .
$$

Now Mr. M. Kuranishi raised the following problem: when $f$ defines an associative composition for every group $G$ ?

We shall solve this problem in this note (Proposition 1), and determine moreover associative compositions holding for all finite nilpotent groups using a theorem of Prof. K. Iwasawa" (Proposition 2). This result will be refined by Proposition 3.

PROPOSITION 1. In order that $f(X, Y)$ define an associative composition for a free group $F_{2}$ on two generators, it is necessary and sufficient that $f$ is one of the following five types:

$$
1, X, Y, X Y, Y X \text {. }
$$

Proof. An element $t \neq 1$ of a free group generated by $x$ and $y$ can be expressed uniquely in the form $z_{1}^{\epsilon_{1}} \ldots z_{k}^{e_{k}}$, where every $z_{i}$ is either $x$ or $y$, where $z_{i} \neq z_{i+1}$ and where $e$ 's are non-vanishing integers. $k$ is called the length of $t$, and is denoted by $l(t)$ ( set $l(1)=0)$. Then one will easily verify

$$
l\left(t^{f}\right) \geq l(t), \quad(f \neq 0),
$$

Received March 17, 1954. 
for any word $t$.

Now, let (3) be an associative composition in $F_{2}$, defined by $f$ in (1) such that $n_{1} \neq 0, \ldots, m_{r} \neq 0$. From the associativity

$$
(a \circ e) \circ e=a \circ(e \circ e), \quad(a \neq e),
$$

we deduce at once that

$$
\sum m_{i}=1 \text { or }=0
$$

similarly we have

$$
\sum n_{i}=1 \text { or }=0
$$

Now, we may assume $m_{1} \neq 0$, since a new composition $a * b=b \circ a$ is associative at the same time as $a \circ b$, and then we have only to prove $r=1$. Suppose $r \gtrsim 2$, and compare two expressions

$$
\begin{aligned}
& (a \circ b) \circ c=(a \circ b)^{m_{1}} c^{n_{1}} \ldots, \\
& a \circ(b \circ c)=\left\{\begin{array}{lll}
a^{m_{1}} b^{m_{1}} c^{n_{1}} \ldots, & \text { if } & n_{1}>0, \\
a^{m_{1}} c^{-n_{r}} b^{-m_{r}} c^{-n_{r-1}} \ldots, & \text { if } & n_{1}<0,
\end{array}\right.
\end{aligned}
$$

for $a, b, c \in F$. If we take $a, b, c$ satisfying no non-trivial relation among themselves (e.g. $x^{2}, x y, y^{2}$ if $F_{2}$ is generated by $x$ and $y$ ), it follows that the length of $(a \circ b)^{m_{1}}$, as an element of the free group generated by $a$ and $b$, is at most 2. But this is the case only if the length of $a \circ b$ itself is at most 2 by (5), contradicting the assumption $r \geqslant 2$. Hence we must have $r=1$. q.e.d

Proposition 2. If $f(X, Y)$ defines an associative composition for every finite nilpotent group generated by two elements, then $f(X, Y)$ is one of the following five types:

$$
1, X, Y, X Y, Y X \text {. }
$$

Proof. Let $F_{2}$ be a free group on two generators $x, y$. By a theorem of K. Iwasawa ${ }^{1)}$ the intersection of all normal subgroups $N$ in $F_{2}$ such that $F_{2} / N$ is a finite nilpotent group coincides with the identity group:

$$
\cap N=\{1\} \text {. }
$$

Now, since $f(X, Y)=X \circ Y$ defines an associative composition for $F_{2} / N$, we

1) K. Iwasawa, Einige Sätze über freie Gruppen, Proc. Imp. Acad. Japan, 19 (1943), pp. $272-274$ 
have for every element $z_{1}, z_{2}, z_{3}$ in $F_{2}$

$$
\left(z_{1} \circ z_{2}\right) \circ z_{3} \equiv z_{1} \circ\left(z_{2} \circ z_{3}\right) \quad(\bmod . N)
$$

Hence we have by (6)

$$
\left(z_{1} \circ z_{2}\right) \circ z_{3}=z_{1} \circ\left(z_{2} \circ z_{3}\right) .
$$

Thus the proposition follows from Proposition 1.

Now we can refine Proposition 2 as follows:

Proposition 3. Let $p>0$ be a given prime integer. If $f(X, Y)$ defines an associative composition for every finite p-group generated by two elements, then, $f(X, Y)$ is one of the following five types

$$
1, X, Y, X Y, Y X
$$

Proof. It is sufficient to show that the intersection of all normal subgroups $M$ in $F$ (a free group on two generators) such that $F / M$ is a finite $p$-group coincides with the identity group:

$$
\cap M=\{1\} .
$$

This fact can be proved quite similarly as in K. Iwasawa ${ }^{1)}$ and we shall show only the corresponding lemma and theorem.

Let $G$ be an arbitrary finitely generated group and

$$
G=Z_{1} \supset Z_{2} \supset \ldots
$$

be the descending central series of $G$, i.e. $Z_{i+1}$ be the subgroup of $G$ generated by $\left(g, z_{i}\right)=g z_{i} g^{-1} z_{i}^{-1}\left(g \in G, z_{i} \in Z_{i}\right)$ :

$$
Z_{i+1}=\left(G, Z_{i}\right) \quad(i=1,2, \ldots)
$$

Then, as is seen easily, ${ }^{2)} Z_{i} / Z_{i+1}$ is a finitely generated abelian group and the torsion of $Z_{i} / Z_{i+1}$ (i.e. the subgroup formed by all elements in $Z_{i} / Z_{i+1}$ which are of finite order) is a finite group.

Now let us call a finitely generated group $G$ to be of p-type if every torsion of $Z_{i} / Z_{i+1}$ is a finite $p$-group. $(i=1,2, \ldots)$

Then an analogy of "Satz 1 " in K. Iwasawa ${ }^{1)}$ is given by

2) Note that $Z_{i} / Z_{i+1}$ is a central subgroup of $G / Z_{i+1}$. Then for every $a, b$ in $G, c, d$ in $Z_{i-1}$ we have $(a b, c d) \equiv(a, c) \cdot(a, d) \bullet(b, c) \bullet(b, d)$ (mod. $\left.Z_{i+1}\right)$ (cf. H. Zassenhaus, Lehrbuch der Gruppentheorie, S. 57). The assertion is then completed by induction on $i$. 
TheOREM. Let $G$ be a finitely generated nilpotent group of p-type. Then the intersection of all normal subgroups $M$ in $G$ such that $G / M$ is a finite $p$ group coincides with the identity group:

$$
\cap M=\{1\} .
$$

This theoren can be proved quite similarly as in K. Iwasawa, l. c. using the following lemma which is a direct corollary of his "Hilfssatz."

LeMmA. Let $G$ be an arbitrary group and let $N$ be a normal subgroup with finitely many generators $a_{1}, \ldots, a_{r}$ such that $(G, N)$ is a central, finite subgroup in $G$ of order $l=p^{\nu}$. Then the subgroup $M$ of $G$ generated by finitely many elements $a_{1}^{l}, \ldots, a_{r}^{l}$ and $(G, N)$ is a central subgroup of $G$ and the factor group $N / M$ is a finite p-group.

Now in order to prove (7) it is sufficient to show that $F / F^{(n)}$ is a group of $p$-type, where $F=F^{(1)}, F^{(i+1)}=\left(F, F^{(i)}\right)(i=1,2, \ldots)$. However, as is wellknown, $F^{(i)} / F^{(i+1)}$ is a free abelian group ${ }^{3)}$ (with finitely many generators). Hence $F / F^{(n)}$ is of $p$-type $(n=1,2, \ldots)$. Thus Proposition 3 is proved.

3! Cf. E. Witt, Treue Darstellung Liescher Ringe, Crelle 177, (1937). 